\title{
Euphrasia cisalpina Pugsley (Orobanchaceae) nella Brughiera di Gallarate (Lombardia, Italia): dati storici e conferma della stazione nelle aree verdi dell'Aeroporto di Milano Malpensa
}

\section{Marco Martignoni}

Via del Lavoro 3, I-21050 Lonate Ceppino (VA), Italia. E-mail: marcomartignoni1974@libero.it

\begin{abstract}
Riassunto - L'autore riporta i dati storici dei rinvenimenti di Euphrasia cisalpina Pugsley, stenoendemita insubrico occidentale, nella Brughiera di Gallarate o "Brughiera Grande" e suoi dintorni, segnalando l'attuale persistenza di una consistente popolazione all'interno dell'Aeroporto di Milano Malpensa, l'unica sopravissuta nell'area. La stazione interessa un'ampia superficie relitta di brughiera pedemontana, situata su un paleogreto del fiume Ticino tra 209 e 237 $\mathrm{m}$ s.l.m. La popolazione sembra trarre giovamento dalla gestione aeroportuale delle aree verdi.
\end{abstract}

Abstract - Euphrasia cisalpina Pugsley (Orobanchaceae) in Gallarate Heathland (Lombardy, Italy): historical data and confirmation of the station in the green areas of the Milano Malpensa Airport.

The author reports the historical data of the presence of Euphrasia cisalpina Pugsley (Orobanchaceae), a western insubric stenoendemite, in Gallarate Heathland (Lombardy, Italy) and surroundings, confirming the presence of a large population, the only survived in the area, in the green space of the Milano Malpensa Airport. The site includes a large relict foothill heathland on a palaeochannel of Ticino river, between 209 and $237 \mathrm{~m}$ a.s.1. The population seems to benefit from the management of airportual green areas.

Key-words: Euphrasia cisalpina, Gallarate Heathland, Milano Malpensa Airport.

\section{INTRODUZIONE}

Nel presente lavoro viene discusso il ritrovamento di una consistente popolazione di Euphrasia cisalpina Pugsley (eufrasia del Ticino; Fig. 1) nella brughiera conservatasi all'interno dell'Aeroporto di Milano Malpensa. Le osservazioni sul campo includono un periodo pressochè continuativo dall'agosto 2010 al luglio 2014 durante il quale sono stati regolarmente rinvenuti numerosi esemplari di E. cisalpina. La specie, ancor oggi poco conosciuta dai botanici italiani (Pignatti, 1982, ne mette addirittura in dubbio la presenza in Italia e la consistenza del rango specifico), è stata segnalata l'ultima volta nell'area della brughiera gallaratese da Giacomini (1958). Vengono inoltre analizzati i dati bibliografici e i campioni d'erbario storici relativi alla specie nell'area gallaratese e brevemente discussi gli interventi gestionali che sembrano favorire la conservazione della popolazione studiata.
Il genere Euphrasia è uno dei più critici della flora d'Italia con forme stagionali ed ecotipiche, caratteri diagnostici poco accentuati, tendenza all'ibridazione e alle mutazioni (Yeo, 1968; Pignatti, 1982; Vitek, 2009). Non stupisce dunque che Euphrasia cisalpina venga spesso confusa con altre specie simili e, di conseguenza, risulti poco segnalata. Descritta per la prima volta da Pugsley (1932a, 1932b), rimane una delle eufrasie meno studiate: le citazioni bibliografiche e i campioni d'erbario di questa specie infatti sono così sporadici, che si è cercato di includere nel presente lavoro tutti quelli relativi all'area gallaratese. Il suo areale si limita a poche località accertate nei Cantoni Ticino (che dà il nome volgare alla specie) e Grigioni (Svizzera), Lombardia e Piemonte (Italia). È inclusa nell'elenco delle specie di flora spontanea protette in modo rigoroso in Lombardia ai sensi della Legge Regionale 31 marzo 2008, n. 10 "Disposizioni per la tutela e la conservazione della piccola fauna, della flora e della vegetazione spontanea" e successiva Deliberazione Giunta Regionale Lombardia 24 luglio 2008, n. 8/7736 (Regione Lombardia, 2010).

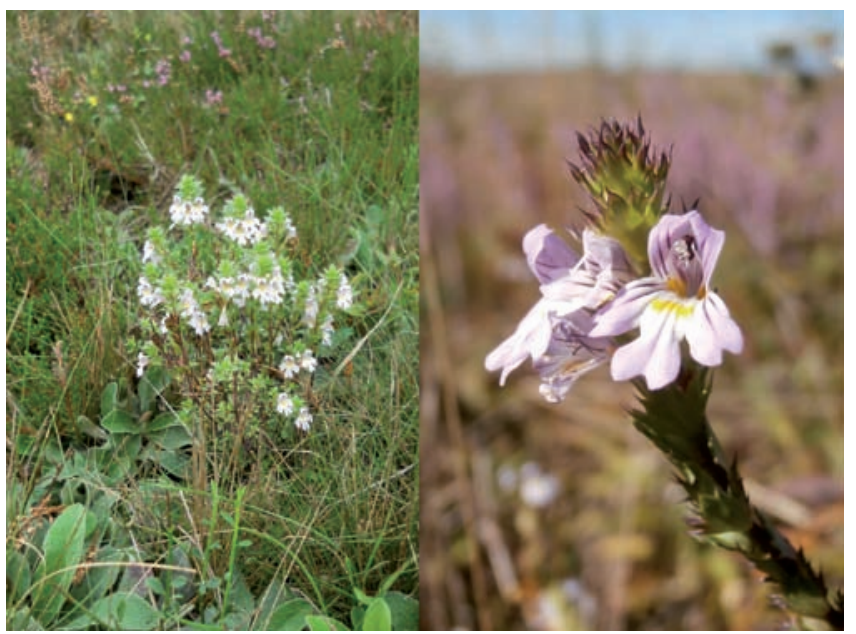

Fig. 1 - Euphrasia cisalpina Pugsley (eufrasia del Ticino / Tessin Eyebright) all'Aeroporto di Milano Malpensa / in the Milano Malpensa Airport. (Foto / Photo M. Martignoni; a sinistra 9 ottobre 2010, a destra 19 settembre 2013 / left $9^{\text {th }}$ October 2010, right $19^{\text {th }}$ September 2013). 


\section{AREA DI STUDIO}

Per Brughiera di Gallarate (in dialetto locale "brüghéra de Galaràa", Cherubini, 1839) o "Brughiera Grande" s'intende un'ampia area di "incolti" che un tempo si estendeva su un terrazzamento a est del fiume Ticino, a sua volta residuo di un grande altipiano diluviale che orlava ininterrottamente il margine meridionale della Prealpi, ora ridotta a miseri lembi relitti e degradati. Essa poggia su spessi depositi fluvioglaciali sabbioso-ciottolosi (Würm) nei quali sono rappresentate tutte le rocce della "serie dei Laghi". Il substrato risulta quindi molto drenante e, di conseguenza, arido (aridità edifica). Dal punto di vista floristico la brughiera gallaratese vanta entità botaniche psammo-litofile e xerofile (Giacomini, 1958; Andreis \& Cerabolini, 1993), pressoché assenti o poco rappresentate nelle altre brughiere pedemontane dell'Italia nordoccidentale (vaude e baraggie del Piemonte, groane della Lombardia), dalle quali sembra differenziarsi in modo sensibile tanto da poter essere considerata come una variante unica. Essa risulta inoltre, almeno fisionomicamente, analoga alle brughiere dei substrati sabbiosi d'oltralpe, come la Lüneburger Heide della Germania settentrionale $\mathrm{e}$, in generale, alle brughiere dell'Europa occidentale a clima oceanico (Brizi \& Fenaroli, 1927; Giacomini, 1958;
Giacomini \& Fenaroli, 1958). Le brughiere pedemontane, ben note per la loro sterilità, sono state oggetto di numerosi tentativi di forestazione e bonifica sin dalla seconda metà del XVIII secolo (Sulli, 1985; Sulli \& Zanzi-Sulli, 1994). Carlo Cozzi (Bubbiano, 1871 - San Macario di Samarate, 1945), sacerdote e appassionato di botanica, già nel primo decennio del secolo scorso avvertiva della grave minaccia che incombeva sulla flora dei terrazzi del Ticino, da lui considerati "una vera oasi floristica" rispetto alla pianura sottostante, minaccia allora costituita principalmente, a suo dire, dalle bonifiche agricole, dalla raccolta di alcune specie per usi medicinali od ornamentali, dall'invasività di qualche specie esotica e dalle prime concimazioni chimiche con l'utilizzo di sali (Cozzi, 1909, 1912). Cozzi fu dunque conservazionista ante litteram e in controtendenza per l'epoca, se si pensa che negli anni venti del secolo scorso vennero pubblicati libri dal titolo "Appunti sul problema della brughiera" (Pratolongo, 1923) e che, nel secondo dopoguerra, nacque 1'“Associazione Forestale Lombarda per la redenzione delle terre incolte e per l'incremento della selvicoltura". Tra gli ultimi lembi relitti di brughiera gallaratese, quello dell'Aeroporto di Milano Malpensa risulta fisionomicamente e floristicamente tra $\mathrm{i}$ più conservati, se non il migliore (Fig. 2). Sono infatti pre-

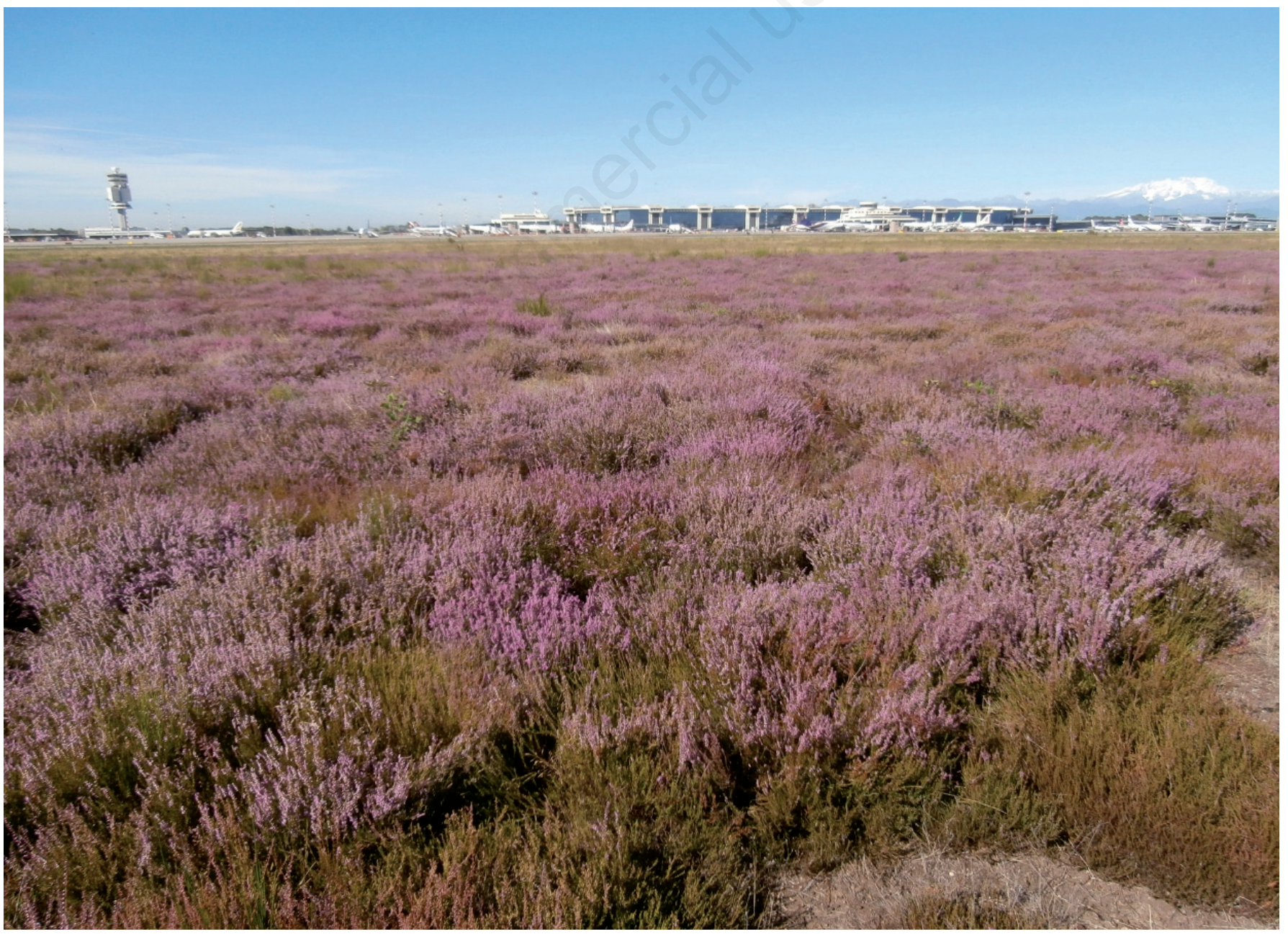

Fig. 2 - Vegetazione di brughiera all'interno dell'Aeroporto di Milano Malpensa. / Heathland in the Milano Malpensa Airport. (Foto / Photo M. Martignoni, 19 Settembre $2013 / 19^{\text {th }}$ September 2013). 
senti e ottimamente rappresentati i seguenti aspetti della brughiera gallaratese descritti originariamente da Giacomini (1958): "gramineto a Molinia altissima", "calluneto molinietoso", "calluneto ottimale", "calluneto lichenoso" e "pelouse xerofila". Il motivo principale risiede nel fatto che la destinazione aeroportuale ha sottratto tali aree alla minaccia delle bonifiche agricolo-forestali, dell'espansione urbanistico-industriale, dell'attività estrattiva e della colonizzazione da parte di essenze arboree ed arbustive, in particolare quelle alloctone, quest'ultima ostacolata dall'oculata gestione a sfalci periodici praticata nelle aree aeroportuali. Non bisogna infatti dimenticare che anche l'assenza di gestione può portare alla scomparsa di habitat particolari: già Giacomini (1958) e Giacomini \& Fenaroli (1958) affermavano che la pratica della rimozione di brugo per farne strame era un'attività umana che favoriva la conservazione delle varie facies della brughiera. Anche il parziale abbandono della tradizionale gestione forestale, mediante taglio periodico degli alberi per ricavarne legname, è causa di un'eccessivo imboschimento ed aduggiamento delle brughiere con conseguente impoverimento floristico. In sostanza, in assenza gestione, la brughiera è destinata all'evoluzione, in tempi più o meno lunghi, verso climax forestali floristicamente meno biodiversificati. È quindi probabile che la gestione delle aree verdi di Milano Malpensa con sfalci periodici, non troppo frequenti e differenziati per zone e ambienti, ricopra una funzione simile alle tradizionali pratiche antropiche, evitando sia la crescita di piante arboree e alto-arbustive sia l'eccessivo sviluppo (in fittezza, altezza ed estensione) della copertura erbacea (molinieti) e microarbustiva (calluneti) che finirebbero per banalizzare la brughiera. In questo modo vengono infatti mantenuti nel tempo habitat a maggiore biodiversità e vengono favorite le specie eliofile, destinate a scomparire in ambienti forestali troppo ombrosi, come le pinete di brughiera o, peggio, $i$ boschi degradati a dominanza di piante legnose alloctone invasive come Prunus serotina Ehrh. (prugnolo tardivo) e Robinia pseudoacacia L. (robinia). Euphrasia cisalpina sembra infatti una specie eliofila, che nei lembi di brughiera esterni all'aeroporto è scomparsa quasi sicuramente per i suddetti motivi, che hanno portato alla degradazione del suo habitat naturale. La vegetazione all'interno dell'aeroporto risulta così ben conservata che si possono ancora ritrovare gran parte delle specie elencate nei rilievi fitosociologici di Giacomini (1958). Sono attualmente in corso uno studio dedicato alla flora e vegetazione dell'Aeroporto di Milano Malpensa e una revisione della distribuzione di Euphrasia cisalpina in Italia, con lo scopo di valutarne lo stato di conservazione a livello nazionale.

\section{RISULTATI}

Dalle osservazioni sul campo (agosto 2010 - luglio 2014) si conferma la presenza di una consistente popolazione di Euphrasia cisalpina all'interno dell'Aeroporto di Milano Malpensa, nel territorio dei comuni di Cardano al Campo, Casorate Sempione, Ferno, Lonate Pozzolo, Samarate e Somma Lombardo, in provincia di Varese (Fig. $3)$. La specie sembra prediligere gli ambienti costituiti da calluneti radi e da pelouse xerofila di brughiera (Fig. 4). La

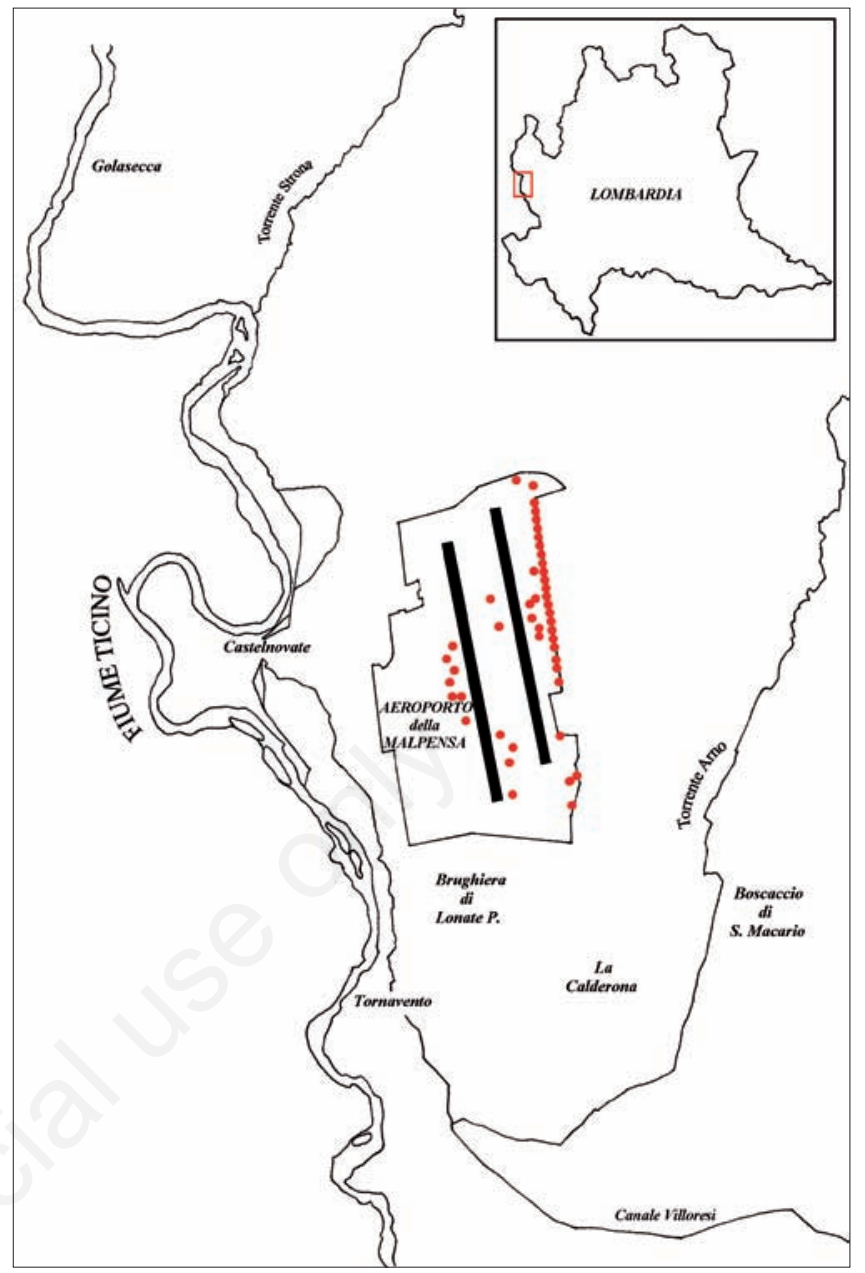

Fig. 3 - Carta dell'area gallaratese con idrografia semplificata e indicazione dei toponimi citati nel testo. $\bullet$ : recenti rinvenimenti di $E$. cisalpina all'interno dell'Aeroporto di Milano Malpensa; le barre nere indicano le piste di volo. / Map of the Gallarate area with simplified hydrography and indication of the toponyms cited in the text. $\bullet$ : recent records of E. cisalpina in the Milano Malpensa Airport; the black bars are the runways.

minaccia principale alla sua conservazione consiste nella colonizzazione da parte di arbusti (come Cytisus scoparius (L.) Link e Rubus spp.) e alberi (in particolare Prunus serotina) e nell'eccessivo sviluppo in densità e altezza dei calluneti (vegetazioni a dominanza di brugo, Calluna vulgaris (L.) Hull.) e dei molinieti (gramineti a Molinia arundinacea Schrank), minaccia che viene scongiurata dalla gestione aeroportuale delle aree verdi. In base alle osservazioni sembra infatti che Euphrasia cisalpina preferisca le aree sfalciate frequentemente (con periodicità variabile a seconda dei tipi di vegetazione) e che l'utilizzo di trinciatrici ne favorisca la disseminazione. La sua ricerca nei lembi di brughiera esterni all'aeroporto ha dato risultati negativi ed è dunque da ritenersi estinta nell'area gallaratese (intesa in senso lato, abbracciando di fatto l'alta pianura ghiaiosa e i terrazzi alluvionali del fiume Ticino e dei torrenti Strona e Arno) al di fuori del sedime aeroportuale. La presente segnalazione costituisce la prima conferma della specie in tempi recenti a livello regionale. 


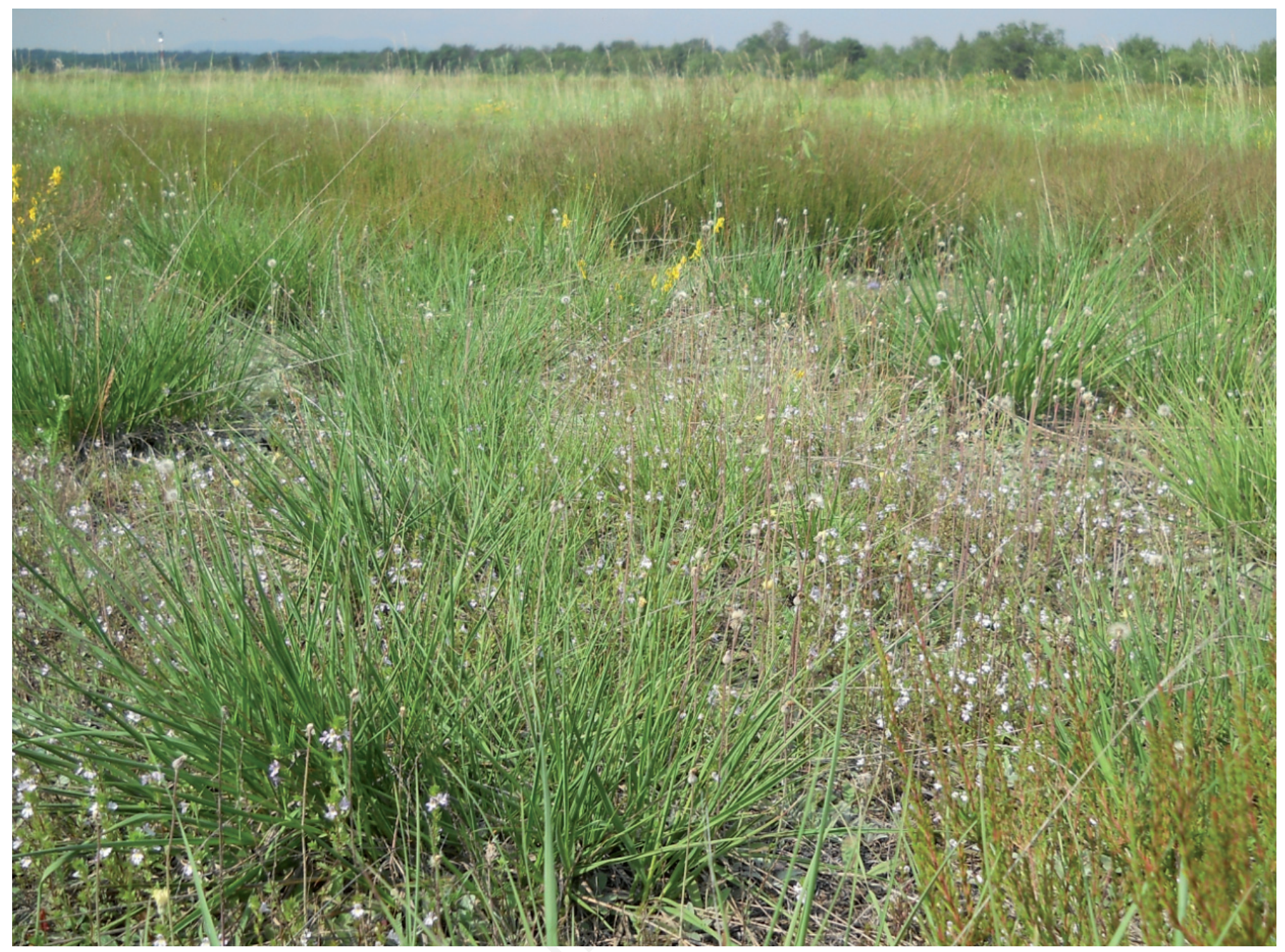

Fig. 4 - Popolamento di Euphrasia cisalpina nella brughiera rada dell'Aeroporto di Milano Malpensa. / Euphrasia cisalpina population in the sparse heathland of Milano Malpensa Airport. (Foto / Photo M. Martignoni, 14 giugno $2012 / 14^{\text {th }}$ June 2012).

Dalle fonti bibliografiche botaniche del XIX secolo non possiamo che dedurre poche notizie: la tassonomia del genere Euphrasia risultava allora molto semplificata e occorrerà attendere gli anni ' 30 del $\mathrm{XX}$ secolo per la descrizione di questa specie da parte di Pugsley (1932a; 1932b). I suoi campioni venivano precedentemente indicati con altri binomi, come E. officinalis L., E. salisburgensis Funk ex Hoppe, E. stricta D.Wolff ex J.F.Lehm., E. nemorosa (Pers.) Wallr., quest'ultima da escludere dall'Italia (Pignatti, 1982). Le opere botaniche dell'epoca riportano inoltre indicazioni geografiche troppo ampie per essere utilizzate nella ricostruzione dell'areale di uno stenoendemita. Per segnalazioni maggiormente circoscritte alla brughiera gallaratese occorre attendere le osservazioni del già citato Carlo Cozzi, sacerdote dal 1909 al 1945 presso la Cascina Sopra di San Macario (Samarate, VA). A lui si devono infatti le prime segnalazioni documentate di piante del genere $E \mathbf{~}$ phrasia nell'area gallaratese: Euphrasia officinalis "Molto comune in tutti i nostri boschi. Non vi ho mai rinvenuto la salisburgensis." (Cozzi, 1902); Euphrasia nemorosa " tra le specie boschive", "quelle cioè caratteristiche della macchia e dell'ericeto" presso Golasecca (Cozzi, 1913); Euphrasia officinalis L. tra le piante che gli erborai "raccoglieva- no, per se o per incarico d'altri, nei paraggi in discorso" (Cozzi, 1912); il fungo parassita Coleosporium euphrasiae (Schumach.) G.Winter, oggi C. tussilaginis (Pers.) Lév.,"su Euphrasia officinalis, nella brughiera del Ticino" (Cozzi, 1917). Le indicazioni di Cozzi per E. officinalis e E. nemorosa corrispondono quasi sicuramente a Euphrasia cisalpina; del resto i campioni dell'erbario Cozzi (ora custodito presso l'Associazione Antares di Legnano) (Antares Legnano, 2008; Anonimo, 2008) raccolti "al Boscaccio di S. Macario" (Samarate, VA) sono certamente attribuibili a quest'ultima specie.

Nel 1927 in un'esaustiva monografia sulle brughiere (Brizi \& Fenaroli, 1927) compare, tra le specie delle brughiere lombarde, E. rostkoviana Hayne "nella brughiera di Lonate Pozzolo (Molinieto-Robinietum)" (pag. 64), tra "gli elementi caratteristici e costanti della brughiera lombarda" (pag. 65), associata al "Molinietum" (pag. 68) e "frequente e diffusa in tutta la brughiera" (pag. 88). In questo volume viene riportato anche un dato di Cozzi per E. nemorosa L. "rara a Castelnovate (Cozzi)" (pag. 88) per il quale non è stato possibile rintracciare ulteriore documentazione bibliografica o campioni d'erbario collegati e quindi, probabilmente, da riferirsi ad una comunicazione personale del 
Cozzi. Anche in questo caso, in attesa di eventuali riscontri d'erbario collegati, si presume che tutte le eufrasie di brughiera citate debbano attribuirsi a Euphrasia cisalpina, l'unica specie sinora rinvenuta in quest'area.

Un altro appassionato di botanica nell'area fu Carlo Stucchi (Milano, 1894 - Cuggiono, 1975), medico condotto di Cuggiono (MI). Egli raccoglieva eufrasie nella brughiera gallaratese già nel 1911 (MSNM-2144), ma le sue prime segnalazioni scritte risalgono al 1929 sub " $E$. salisburgensis Funck - Qua e là nelle brughiere" (Stucchi, 1929). Si deve proprio a Stucchi l'aver intuito e verificato, molti anni dopo, che le eufrasie presenti nella brughiera gallaratese dovevano identificarsi con la specie descritta da Pugsley nel 1932, avvalendosi della flora del Binz (1949), dell'aiuto del botanico svizzero Hans Dübi (Berna, 1881 - Cortivallo di Lugano, 1968) e di confronti con campioni d'erbario provenienti dal Canton Ticino (MSNM-2146; TR) (Stucchi, 1956).

In quest'area erborizzò anche Paola Zaro (1905-1989), maestra elementare a Lonate Pozzolo (VA), che conobbe sia Carlo Cozzi che Carlo Stucchi e con i quali era in contatto. Nel suo erbario, custodito presso il Comune di Samarate (VA), compaiono due campioni del 1948, rispettivamente sub E. officinalis L. ssp. salisburgensis Hoppe e sub E. stricta Hoppe, raccolte entrambe "alla Calderona" (Lonate Pozzolo, VA). Anche questi esemplari sono da attribuirsi a E. cisalpina.

Il 26 luglio 1957 la brughiera gallaratese riceve anche le attenzioni della terza escursione fitosociologica internazionale: dai rilievi riportati nella relazione di Giacomini (1958) troviamo E. cisalpina "presso Tornavento (Gallarate) (terreno pianeggiante, $100 \mathrm{~m}^{2}$, riferibile al «Calluneto lichenoso»"), nonché nella "pelouse xerofila": "Brughiera presso Novate al margine della strada, a m 203 ca." (Castelnovate, Vizzola Ticino, VA).

Nell'erbario Fenaroli (conservato presso il Museo Tridentino di Scienze Naturali di Trento) è presente un campione di Euphrasia cisalpina raccolto nella brughiera gallaratese da Stucchi e accompagnato da una sua lettera. Fenaroli dovette probabilmente convincersi che le eufrasie di brughiera, in precedenza attribuite a E. rostkoviana da Brizi \& Fenaroli (1927), erano in realtà da ricondurre a E. cisalpina giacché nel suo erbario vi è un altro campione, raccolto nel luglio 1957 nella brughiera gallaratese dallo stesso Fenaroli (forse proprio in occasione della terza escursione fitosociologica internazionale), etichettato come E. cisalpina.

Nei primi sessant'anni del secolo scorso (1902-1957) la brughiera gallaratese fu dunque discretamente frequentata da botanici e appassionati ed E. cisalpina era frequente e veniva regolarmente rinvenuta. Da allora non è stata più osservata, sia a causa della cessazione delle attività antropiche tradizionali sia dell'ampliamento dell'aeroporto, che ha interdetto il libero accesso alle aree pur conservandone sostanzialmente gli ambienti naturali.

Per completezza va citata anche la tesi della dr.ssa Adriana Noto (a.a. 1968-1969), il cui relatore fu Valerio Giacomini. In una copia autografata dalla Noto e donata a Carlo Stucchi, ora conservata nella biblioteca del Museo di Storia Naturale di Milano, compare E. rostkoviana "tra gli elementi caratteristici e costanti della brughiera lom- barda" (pag. 36), con una correzione a mano: "? NO; $E$. stricta". A pagina 68 della stessa tesi troviamo invece $E$. cisalpina "E l'euphrasia comune nella brughiera in senso stretto". In mancanza di altre indicazioni si deve supporre che tale tesi sia di carattere prettamente compilativo e che le segnalazioni di eufrasie in essa contenute siano riprese dalle precedenti fonti bibliografiche, rispettivamente Brizi \& Fenaroli (1927) per E. rostkoviana e Giacomini (1958) per E. cisalpina. Se ne deduce che l'ultimo dato reale di E. cisalpina nella Brughiera di Gallarate è quello di Giacomini (1958).

Recentemente E. cisalpina viene citata come "presente", ma soltanto su base bibliografica, in Lombardia (Banfi \& Galasso, 2005) e "da ricercare", "da confermare" o "da verificarne la presenza" in provincia di Varese (rispettivamente in: Macchi, 2005; Bogliani et al., 2007; Regione Lombardia, 2010).

\section{CONCLUSIONI}

La stazione qui descritta, relativa all'Aeroporto di Milano Malpensa, conferma la presenza della specie nell'area gallaratese dopo oltre 50 anni dall'ultimo ritrovamento documentato (Giacomini, 1958). Sebbene il sito non sia sottoposto a nessun vincolo ambientale, essendo incluso all'interno del sedime aeroportuale di Malpensa, attualmente non sussistono pericoli per la popolazione in questione in quanto la gestione delle aree verdi all'interno dello scalo sembra favorire la permanenza della specie altrove scomparsa a causa di numerosi fattori antropici come l'espansione urbanistico-industriale, le attività estrattive, le bonifiche agricolo-forestali, l'abbandono della gestione tradizionale delle brughiere e delle selve e l'eccessiva colonizzazione da parte di essenze arboree ed arbustive.

\section{Specimina visa}

Brughiera di Gallarate, Sep 1911, C. Stucchi (MSNM-2144, ex Hb. Stucchi); Boscaccio di S. Macario, Jun 1912, C. Cozzi (Herbarium C. Cozzi, presso Associazione Antares di Legnano, sub Euphrasia officinalis L.); Brughiera di Gallarate, Sep 1929, C. Stucchi (MSNM-2145, ex Hb. Stucchi); Lombardia: nella brughiera di Gallarate (Varese), Sep 1929, C. Stucchi (TR, ex $\mathrm{Hb}$. Fenaroli, ex Hb. Stucchi; allegata una lettera di C. Stucchi a L. Fenaroli); Sopra Breno, Malcantone. (C. Ticino) Nelle selve di Castagno, Aug 1945, H. Dübi (MSNM-2146, ex Hb. Stucchi); Svizzera: nel Canton Ticino, sopra Breno (Malcantone) nei castagneti, Aug 1945, C. Stucchi?, H. Dübi? (TR, ex Hb. Fenaroli, ex Hb. Stucchi); Boschi Calderona, May 1948, P. Zaro (Herbarium Zaro, presso Comune di Samarate, sub Euphrasia stricta Hoppe); Brughiere della Calderona, Jul 1948, P. Zaro (Herbarium Zaro, presso Comune di Samarate, sub Euphrasia officinalis L. ssp. salisburgensis Hoppe); Lombardia: nell'Alto Milanese, nella brughiera di Lonate-Pozzolo, Jul 1957, L. Fenaroli (TR, ex Hb. Fenaroli); Italien lombardei, Heide bei Gallarate, 26 Jul 1957, E. Furrer, (ZT-36807); Brughiera di Gallarate, Sep 1957, C. Stucchi (MSNM-2132, ex Hb. Stucchi); Brughiera di Gallarate, Sep 1957, C. Stucchi (MSNM-8716, ex Hb. 
Stucchi); Aeroporto di Milano Malpensa, Aug 2010, M. Martignoni (MSNM-44904); Aeroporto di Milano Malpensa, 15 Jun 2012, M. Martignoni (MSNM-44905); Aeroporto di Milano Malpensa, 6 Sep 2012, M. Martignoni (MSNM-44906); Aeroporto di Milano Malpensa, 6 Oct 2012, M. Martignoni (MSNM-44907); Aeroporto Milano Malpensa, 13 Jul 2013, M. Martignoni (MSNM-44908) Aeroporto di Milano Malpensa, 20 Sep 2013, M. Martignoni (MSNM-44909).

\section{Ringraziamenti}

Ringrazio tutti coloro che mi hanno incoraggiato e aiutato a ricostruire la storia di Euphrasia cisalpina nel gallaratese: 1'Associazione Antares di Legnano (MI) ed, in particolare, il Sig. Gilberto Oldrini; Grazia Genovese e la dr.ssa Tiziana Primi del Comune di Samarate (VA); Enzo Bona di Capo di Ponte (BS); il dr. Matthias Baltisberger dell'Eidgenössische Technische Hochschule Zürich -Zentrum; il dr. Costantino Bonomi del Museo Tridentino di Scienze Naturali di Trento; il dr. Gabriele Galasso del Museo di Storia Naturale di Milano; il dr. Simone Orsenigo dell'Università degli Studi di Pavia; il Sig. Massimo Antinori di Firenze; la SEA S.p.A. ed, in particolare, la dr.ssa Lucia Giaculli.

\section{BIBLIOGRAFIA}

Andreis C. \& Cerabolini B., 1993 - La Brughiera briantea, la vegetazione ed il piano di gestione. Colloques Phytosociologiques, Vaduz, 21: 195-224.

Anonimo, 2008 - Herbarium Carlo Cozzi. Erbe e fiori di un secolo fa. Associazione Antares, Legnano.

Antares Legnano (ed.), 2008 - Herbarium Carlo Cozzi. Freeman editrice, Busto Arsizio.

Banfi E. \& Galasso G., 2005 - Lombardia. In: An Annotated Checklist of the Italian Vascular Flora. Conti F., Abbate G., Alessandrini A. \& Blasi C. (eds.). Palombi Editori, Roma: 33-185; 237-242.

Binz A., 1949 - Schul- und Exkursionsflora der Schweiz, mit Berücksichtigung der für Basel in Betracht kommenden benachbarten Teile Badens und des Elsasses. B. Schwabe, Basel.

Bogliani G., Agapito Ludovici A., Arduino S., Brambilla M., Casale F., Crovetto G. M., Falco R., Siccardi P. \& Trivellini G., 2007 - Aree prioritarie per la biodiversità nella Pianura Padana lombarda. Fondazione Lombardia per l'Ambiente e Regione Lombardia, Milano.

Brizi U. \& Fenaroli L., 1927 - Appunti sulla flora della brughiera lombarda. In: Le brughiere. Luzzato F., Artini E., Brizi U., Fenaroli L., Pratolongo U., Parisi P., Alpe V. \& Pavari A. (eds.). Federazione Italiana dei Consorzi Agrari, Piacenza: 61-93.

Cherubini F., 1839 - Vocabolario milanese-italiano. Imperial Regia Stamperia, Milano, 1, 2.

Cozzi C., 1902 - Spigolature botaniche nelle brughiere del Ticino. Atti della Società italiana di Scienze naturali e del Museo civico di Storia naturale in Milano, Milano, 41: 427-437.

Cozzi C. 1909 - Sulle variazioni floristiche nei terrazzi del fiume Ticino. Atti della Società italiana di Scienze naturali e del Museo civico di Storia naturale in Milano, Milano, 48: 219-222.

Cozzi C., 1912 - Sulle variazioni floristiche nei terrazzi del fiume Ticino. Nuove osservazioni del socio Sac. Carlo Cozzi. Atti della Società italiana di Scienze naturali e del Museo civico di Storia naturale in Milano, Milano, 51: 68-72.

Cozzi C., 1913 - Erborizzazioni nel morenico di Golasecca. Atti della Società italiana di Scienze naturali e del Museo civico di Storia naturale in Milano, Milano, 52: 306-308.

Cozzi C., 1917 - Manipolo di funghi della pianura milanese. Bullettino della Società Botanica Italiana, Firenze, 24: 94-99.

Giacomini V., 1958 - Sulla vegetazione della Brughiera di Gallarate. In: Relazione sulla terza escursione fitosociologica internazionale (Pavia, 21-26 luglio 1957). Pignatti Wikus E. \& Pignatti S. (eds.). Archivio Botanico e Biogeografico Italiano, Forlì, s. 4, 34, 3 (1-2): 63-68.

Giacomini V. \& Fenaroli L., 1958 - Conosci l'Italia .Volume 2. La Flora, Touring Club Italiano, Milano.

Macchi P., 2005 - La flora della provincia di Varese. Catalogo delle piante vascolari. Provincia di Varese, Varese.

Noto A., 1968-1969 - La brughiera lombarda. Tesi di Laurea inedita, a.a.1968-69. Università degli Studi di Roma, Facoltà di Scienze Matematiche, fisiche e naturali, Roma.

Pignatti S., 1982 - La Flora d'Italia. Edagricole, Bologna, 2.

Pratolongo U., 1923 - Appunti sul problema della brughiera. Tipografia Federazione Italiana Consorzi Agrari, Piacenza.

Pugsley H. W., 1932a - Notes on Euphrasia. Journal of Botany, London, 70: 200-204.

Pugsley H. W., 1932b - Short note. Euphrasia schinzii (p. 202). Journal of Botany, London, 70: 262.

Regione Lombardia, 2010 - Flora e piccola fauna protette in Lombardia. Centro Flora Autoctona della Regione Lombardia (CFA), Galbiate (Lecco).

Stucchi C., 1929 - Note su alcune piante raccolte lungo il Ticino. Nuovo Giornale Botanico Italiano, Firenze, n.s. 36 (1): 34-45.

Stucchi C., 1956 - Piante critiche di Lombardia. Nuovo Giornale Botanico Italiano, Firenze, 62 (1955. 1-2): 356-362.

Sulli M., 1985 - Boschi e brughiere dell'Altopiano milanese: duecento anni di dibattito. Annali dell'Istituto Sperimentale per la Selvicoltura, Arezzo, 16: 313371.

Sulli M. \& Zanzi-Sulli A., 1994 - Da brughiera a bosco: l'Altopiano milanese dalla fine del settecento a oggi. Storia Urbana, Milano, 69:35-72.

Vitek E., 2009 - Euphrasia L. In: Flora Iberica. Plantas vasculares de la Península Ibérica e Islas Baleares. Castroviejo S. (ed.). Real Jardín Botánico, Madrid, 13: 454-473.

Yeo P. F., 1968 - The evolutionary significance of the speciation of Euphrasia in Europe. Evolution, Lawrence, 22 (4): 736-747. 